\title{
Long-term effects of mild traumatic brain injury on cognitive performance
}

\author{
Philip J. A. Dean* and Annette Sterr \\ Department of Psychology, University of Surrey, Guildford, UK
}

\section{Edited by:}

Alvaro Pascual-Leone, Beth Israel Deaconess Medical Center/Harvard Medical School, USA

\section{Reviewed by:}

Nils I. Landro, University of Oslo, Norway

Stefano Sandrone, Vita-Salute San Raffaele University and Institute for Advanced Study IUSS - Pavia, Italy

\section{*Correspondence:}

Philip J. A. Dean, Department of Psychology, University of Surrey, Guildford, GU2 7XH, UK.

e-mail:p.dean@surrey.ac.uk
Although a proportion of individuals report chronic cognitive difficulties after mild traumatic brain injury (mTBI), results from behavioral testing have been inconsistent. In fact, the variability inherent to the $\mathrm{mTBI}$ population may be masking subtle cognitive deficits. We hypothesized that this variability could be reduced by accounting for post-concussion syndrome (PCS) in the sample. Thirty-six participants with $\mathrm{mTBI}$ (>1 year post-injury) and 36 non-head injured controls performed information processing speed (Paced Visual Serial Addition Task, PVSAT) and working memory (n-Back) tasks. Both groups were split by PCS diagnosis (4 groups, all $n=18$ ), with categorization of controls based on symptom report. Participants with $\mathrm{mTBI}$ and persistent PCS had significantly greater error rates on both the n-Back and PVSAT, at every difficulty level except 0-Back (used as a test of performance validity). There was no difference between any of the other groups. Therefore, a cognitive deficit can be observed in mTBI participants, even 1 year after injury. Correlations between cognitive performance and symptoms were only observed for mTBI participants, with worse performance correlating with lower sleep quality, in addition to a medium effect size association (falling short of statistical significance) with higher PCS symptoms, post-traumatic stress disorder (PTSD), and anxiety. These results suggest that the reduction in cognitive performance is not due to greater symptom report itself, but is associated to some extent with the initial injury. Furthermore, the results validate the utility of our participant grouping, and demonstrate its potential to reduce the variability observed in previous studies.

Keywords: head injury, minor, post-concussion syndrome, cognition, neuropsychological tests

\section{INTRODUCTION}

A number of studies report that mild traumatic brain injury (mTBI) participants have reduced cognitive performance, even in the long-term ( $>3$ months) after injury, on tasks that assess attention (Mangels et al., 2002; Chan, 2005; Kumar et al., 2005; Sterr et al., 2006; Catale et al., 2009; Maruta et al., 2010), memory (Vanderploeg et al., 2005; Chen et al., 2007; Catale et al., 2009), executive function (O'Jile et al., 2006; Sterr et al., 2006; Erez et al., 2009; Pontifex et al., 2009), and information processing (O'Jile et al., 2006; Lachapelle et al., 2008; Johansson et al., 2009; Kinnunen et al., 2011). However, there is also research that observes no deficit in cognitive performance in mTBI patients in the long-term (Chen et al., 2004; Perlstein et al., 2004; Solbakk et al., 2005; Broglio et al., 2009; Tellier et al., 2009) or within the 3 months following injury (McAllister et al., 2001; Chen et al., 2004; Jantzen et al., 2004; Lange et al., 2009; Tellier et al., 2009; Slobounov et al., 2010). Indeed, even in those investigations that do detect a deficit, there seems to be little consistency in which cognitive performance is impaired (Tellier et al., 2009). Inconsistency and variability between previous studies on longterm cognitive performance after mTBI is likely to be due to a combination of the variety of tasks used and the distinct samples tested.
A variety of different aspects of cognitive performance have been investigated in the long-term after mTBI, using a number of different tasks. More importantly, tasks assessing the same cognitive function have varied in their difficulty, possibly leading to the inconsistent results. A challenging cognitive task may be required to observe the subtle long-term alterations in participants with mTBI (Segalowitz et al., 2001; Chen et al., 2004). Of particular utility in this regard are tasks that can be parametrically increased in difficulty (Braver et al., 1997; Pare et al., 2009), enabling an investigation of the effects of enhancing cognitive load.

Two tasks that can be parameterized in this way are the n-Back (assessing working memory) and Paced Visual Serial Addition Task [PVSAT, assessing information speed (Fos et al., 2000)]. Both of these tasks have been previously used in mTBI research [ $\mathrm{n}$ Back: (McAllister et al., 1999, 2001; Wang et al., 2006; Catale et al., 2009); PVSAT: (Cicerone and Azulay, 2002; Vanderploeg et al., 2005; O'Jile et al., 2006; Wang et al., 2006; Mayer et al., 2009; Brenner et al., 2010b)], with the paced auditory serial addition task specifically created to investigate cognitive difficulties after TBI. However, few of the previous studies have used a range of difficulties within PVSAT to assess cognition.

In addition, sampling of a mTBI population is challenging, as there is inherent heterogeneity between individuals 
(Shum et al., 2011), with differing severity of injury and subsequent outcome. One way of reducing variability is to split the mTBI sample by post-concussion syndrome (PCS) diagnosis (WHO, 1992), as has been argued previously (Hartlage et al., 2001; Cicerone and Azulay, 2002; Wang et al., 2006). Studies that have split their mTBI sample by PCS diagnosis have been relatively more consistent in their findings of cognitive deficit (Cicerone and Azulay, 2002; Kumar et al., 2005; Sterr et al., 2006; Wang et al., 2006; Chen et al., 2007; Ptito et al., 2007; Johansson et al., 2009).

PCS is the term for the range of cognitive, somatic, and affective symptoms usually reported by participants after a mTBI (Ryan and Warden, 2003). Symptoms typically resolve within 3 months (Korinthenberg et al., 2004; Lundin et al., 2006; Lannsjo et al., 2009; Sigurdardottir et al., 2009; Yang et al., 2009; Sroufe et al., 2010), but in some individuals these symptoms persist (Killam et al., 2005; Sterr et al., 2006; Stulemeijer et al., 2007; Hessen et al., 2008), and can be present years after injury (Vanderploeg et al., 2005). However, PCS symptom report is influenced by depression (Suhr and Gunstad, 2002; Iverson, 2006), chronic pain (Radanov et al., 1992), post-traumatic stress (Hoge et al., 2008; Nelson et al., 2009; Kennedy et al., 2010), anxiety (Moore et al., 2006), fatigue (Johansson et al., 2009), and involvement in litigation (Greiffenstein and Baker, 2001; LeesHaley et al., 2001). Post-concussion-like symptoms have also been reported in healthy participants at levels that would result in PCS diagnosis in a head injured population (Chan, 2001; Iverson and Lange, 2003; Wang et al., 2006; Fear et al., 2009). Furthermore, symptoms such as memory and concentration problems have been shown to emerge during the early recovery phase rather than forming the initial symptom complex (Dikmen et al., 2010; Meares et al., 2011).

Consequently, there is some debate whether persistent PCS (>3 months) is due to biological factors from neural damage or a psychological response to the mTBI (Mittenberg et al., 1992; Bailey et al., 2006; Mulhern and McMillan, 2006). It has been shown that subjective symptom report does not relate to objective symptoms (Nolin et al., 2006; Spencer et al., 2010). This has led some to suggest that PCS is not specific to mTBI (Sroufe et al., 2010), a finding we recently confirmed on a larger sample of 350 participants (Dean et al., 2012). However, the use of adequate control populations can help alleviate some of the problems associated with the non-specificity of PCS. Previous studies have used specific clinical populations such as those with posttraumatic stress disorder (PTSD), chronic pain, and patients with equivalent injuries to the body, sparing the head (Bell et al., 1999; Vanderploeg et al., 2009; Meares et al., 2011). It is also possible to control for post-concussion-like symptoms in healthy participants by splitting this group by PCS in a similar way to those with mTBI. Healthy control participants with levels of symptoms that would result in PCS diagnosis can then be compared to those mTBI participants with PCS. Cognitive differences between these two groups may then be attributed to the report of PCS after mTBI, and not the symptoms alone. Furthermore, if PCS is induced to some extent by damage at the time of injury, then it can be assumed that those mTBI participants with greater symptoms will perform worse on cognitive tasks, whereas there will be no correlation between performance and symptoms in control participants.

Based on the considerations above, the present study investigates working memory and information processing speed in participants a year or more post-mTBI compared to a nonhead injured control population. Both populations were assessed for PCS symptoms, and split into those with and without ongoing PCS to form four participants groups: mTBI + PCS, mTBI - PCS, Control + PCS, and Control - PCS. Control participants are labeled as having PCS when they meet all the DSM-IV criteria (APA, 1994), with the exception of previous head injury.

These four groups were used to test the hypothesis that only participants who report persistent PCS after mTBI will show a cognitive deficit. In contrast, head-injured individuals who report no on-going PCS symptoms, and those without prior head injury (regardless of extent of post-concussion symptoms) are likely to have no evidence of cognitive dysfunction. Furthermore, the cognitive deficit in mTBI participants with PCS will become more apparent as the difficulty of the task is parametrically increased.

\section{MATERIALS AND METHODS PARTICIPANTS \\ Recruitment}

The study specifically aimed to recruit persons who had not sought medical attention following their mTBI. A large number of those who sustain a mTBI are unreported in traditional hospital and emergency department-based research (Segalowitz and Lawson, 1995; NCIPC, 2003; Bazarian et al., 2005). Consequently, participants were recruited from a database generated by a previous study (Dean et al., 2012) which used an online survey aimed at the general public. This survey was open to both those with and without head injury, and recorded demographic information, comprehensive details about any prior head injury (in order to determine whether any injury met the diagnosis criteria for mTBI), and questionnaires on PCS and co-variables as detailed below. Those reporting any form of head injury in the survey were subsequently screened for mTBI according to ICD-10 criteria. The study protocol was given a favorable opinion by the University of Surrey Ethics Committee. Written informed consent was obtained prior to participation.

\section{Diagnosis}

We determined mTBI using ICD-10 criteria (Holm et al., 2005). According to these criteria, participants must report one or more of the following: dizziness or confusion; loss of consciousness $\leq 30 \mathrm{~min}$; post-traumatic amnesia $<24 \mathrm{~h}$. A case history was taken which included a description of the injury, the date of injury, any other head injuries as well as general health and lifestyle information. Only participants at least a year post-mTBI, with no report of litigation, major invasive head injury, chronic pain, or other neurological conditions were contacted to take part in the study. Control participants were selected as those who did not report any prior head injury.

We diagnosed PCS using the modified DSM-IV criteria specified by Mittenberg and Strauman (2000), which requires report 
of three or more of the following symptoms subsequent to head trauma: (1) headache, (2) vertigo or dizziness, (3) irritability or aggression on little or no provocation, (4) anxiety, depression, or affective instability, (5) becoming fatigued easily, (6) disordered sleep, (7) changes in personality, and (8) apathy or lack of spontaneity. The extent of PCS was measured using the Rivermead Post-Concussion symptoms Questionnaire [RPQ; (King et al., 1995)] and Rivermead Post-Concussion symptoms Questionnaire for Controls [RPQ-C; (Sterr et al., 2006; Dean et al., 2012)]. PCS diagnosis was achieved in the same way for control participants as mTBI participants, with the exception that controls had no "history of head trauma." The majority of control participants did not attribute their symptoms to any specific cause, with a few $(n=5)$ attributing them to generalized stress or anxiety.

\section{Study groups}

Once diagnosed, selected participants were then asked to take part in computer-based tasks of memory and mental agility. Four groups with 18 participants each were included in this study (for demographics, see Table 1). The groups were:

- $m T B I+$ PCS: Participants who suffered a mTBI and have persistent PCS

- $m$ TBI - PCS: Participants with mTBI but no current PCS (this does not preclude them having had acute PCS symptoms that have recovered)

- Control + PCS: Participants with PCS, but no history of brain injury

- Control - PCS: Participants with no history of brain injury and no PCS

\section{QUESTIONNAIRES}

In addition to the RPQ, questionnaires that assessed common covariables of PCS were included in the survey (Dean et al., 2012): everyday cognitive failures [Cognitive Failures Questionnaire, CFQ; (Broadbent et al., 1982)], daytime sleepiness [Epworth Sleepiness Scale, ESS; (Johns, 1991)], PTSD [Impact of Event Scale - Revised, IES-R; (Weiss, 2007)], anxiety, and depression [Hospital Anxiety and Depression Scale, HADS; (Bjelland et al., 2002)]. A measure of sleep propensity [Karolinska Sleepiness Scale, KSS; (Gillberg et al., 1994)] was taken before (KSS Pre) and after (KSS Post) the behavioral task. Overall sleep quality [Pittsburgh Sleep Quality Index, PSQI; (Buysse et al., 1989)] and IQ [National Adult Reading Test, NART; (Nelson, 1982)] were assessed either on the day of cognitive testing or on a subsequent day due to time constraints. Therefore, not all participants could complete these two assessments: PSQI (mTBI + PCS: $n=12$; mTBI - PCS: $n=16$; Control + PCS: $n=14$; Control - PCS: $n=13$ ) and NART (mTBI + PCS: $n=14$; mTBI - PCS: $n=17$; Control + PCS: $n=13$; Control - PCS: $n=16$ ).

\section{COGNITIVE TASKS}

Participants were presented with two behavioral tasks: the n-Back and the PVSAT. Both tasks looked identical: single digit numbers between 1 and 9 inclusive were presented on the screen one at a time, with 60 of these stimuli (including 20 randomly ordered target stimuli) per block. There was a total of 12 blocks for each task, with 3 randomly ordered repetitions of the 4 levels of difficulty. The order of presentation (n-Back/PVSAT) was counterbalanced across participants. The keys $\mathrm{M}$ and $\mathrm{C}$ on a standard keyboard were counterbalanced as target and non-target response buttons across the participants.

Table 1 | Demographic and questionnaire data.

\begin{tabular}{|c|c|c|c|c|c|}
\hline & \multicolumn{2}{|c|}{ mTBI } & \multicolumn{2}{|c|}{ Control } & Group difference \\
\hline Gender (F/M) & $12 / 6$ & $10 / 8$ & $10 / 8$ & $10 / 8$ & - \\
\hline IQ (NART) & $112.1 \pm 1.1$ & $116.1 \pm 1.0$ & $115 \pm 1.4$ & $116.0 \pm 1.0$ & - \\
\hline $\mathrm{RPQ}$ & $24.6 \pm 1.8$ & $8.8 \pm 1.5$ & $27.7 \pm 2.5$ & $2.9 \pm 0.7$ & $p<0.001$ \\
\hline HADS: Depression & $4.1 \pm 0.7$ & $2.1 \pm 0.5$ & $5.3 \pm 0.8$ & $2.0 \pm 0.6$ & $p=0.001$ \\
\hline ESS & $8.1 \pm 1.3$ & $6.9 \pm 0.9$ & $8.6 \pm 1.1$ & $5.6 \pm 0.6$ & - \\
\hline KSS: Pre & $3.9 \pm 0.3$ & $4.1 \pm 0.4$ & $4.6 \pm 0.4$ & $3.2 \pm 0.2$ & - \\
\hline KSS: Post & $5.1 \pm 0.4$ & $5.4 \pm 0.4$ & $5.6 \pm 0.4$ & $4.8 \pm 0.4$ & - \\
\hline KSS: Post-Pre & $1.2 \pm 0.4^{*}$ & $1.3 \pm 0.5^{*}$ & $1.0 \pm 0.3^{*}$ & $1.6 \pm 0.5^{*}$ & - \\
\hline
\end{tabular}

Note: All groups: $n=18$, except NART (mTBI + PCS: $n=14 ; m T B I-P C S: n=17 ;$ Control + PCS: $n=13 ;$ Control - PCS: $n=16)$ and PSQI (mTBI+PCS: $n=$ 12; mTBI - PCS: $n=16$; Control + PCS: $n=14$; Control - PCS: $n=13$ ). All figures except Gender expressed as mean \pm SEM. Shaded gray boxes indicate groups generating the significant difference as revealed by Bonferroni-adjusted pairwise comparisons.

${ }^{*}$ KSS Post-significantly greater than KSS Pre in all groups ( $\left.p<0.05\right)$. NART, National Adult Reading Test; RPQ, Rivermead Post-Concussion Symptoms Questionnaire; CFQ, Cognitive Failures Questionnaire; HADS, Hospital Anxiety and Depression Scale; ESS, Epworth Sleepiness Scale; KSS, Karolinska Sleepiness Scale; PSQI, Pittsburgh Sleepiness Index; IES-R, Impact of Event Scale-Revised. 


\section{n-Back}

There were four conditions: 0-Back, 1-Back, 2-Back, and 3-Back. The numbers were presented every $3 \mathrm{~s}$. Participants were asked to press the target button when the number on screen matched the number observed one previous (1-Back), two previous (2-Back), or three previous (3-Back). For every other number that did not match, participants were asked to press the non-target button. In the fourth condition (0-Back) a random number between 1 and 9 was designated as a target at the beginning of the block. Performance on the 0 -Back condition should be near ceiling for all participant groups, and was therefore used as a test of performance validity.

\section{PVSAT}

There were four conditions: $2.5 \mathrm{~s}$ PVSAT, $2 \mathrm{~s}$ PVSAT, $1.5 \mathrm{~s}$ PVSAT, and $1 \mathrm{~s}$ PVSAT. The inter-stimulus interval (ISI) was $2.5 \mathrm{~s}, 2 \mathrm{~s}$, $1.5 \mathrm{~s}$, or $1 \mathrm{~s}$. Each of the four ISI's was presented with each of the three target numbers. Participants were required to add the number on screen to the previously presented number. At the beginning of each block they were given a target number of 9 , 10 , or 11 . If the addition equalled the target number, a "correct" response was required. An "incorrect" response was required for every other addition.

\section{DATA ANALYSIS}

A series of One-Way ANOVAs were carried out on the questionnaire and demographic data (Table 1), with between-subjects factor of GROUP and post-hoc Bonferroni-corrected comparisons. Paired samples $t$-tests were performed for each of the groups to assess the difference between KSS Pre and Post. Gender differences were assessed using a $\chi^{2}$ test.

The cognitive tasks were analyzed using two separate mixedmodel ANOVAs with factor of DIFFICULTY LEVEL (3-, 2-, 1-, 0 -Back or 1, 1.5, 2, $2.5 \mathrm{~s}$ ) and between-subjects factor of GROUP (mTBI + PCS, mTBI - PCS, Control + PCS, Control - PCS), with post-hoc Bonferroni-corrected comparisons as appropriate. Subsequent to this, a series of One-Way ANOVAs were performed for each difficulty level within each task.

In order to investigate the contribution of post-concussion symptoms and its co-variables to cognitive performance after head injury, a series of Spearman's Rho $(\rho)$ correlations were performed. The sample was split into those with mTBI $(n=36)$ and those without $(n=36)$, and average error rates across conditions were calculated as a measure of global performance (n-Back average did not include 0 -Back). Only those co-variables which significantly differed between groups were used in the analysis. Correction for multiple comparisons was used, with a modified threshold $p$-value of 0.002 .

\section{RESULTS}

\section{DEMOGRAPHICS AND QUESTIONNAIRE MEASURES}

There was no significant difference between the groups on any of the demographic data (age, gender, IQ). However, a significant effect of GROUP was observed for post-concussion symptoms [RPQ; $F_{(3,68)}=47.8, p<0.001$ ], cognitive failures [CFQ; $\left.F_{(3,68)}=12.7, p<0.001\right]$, anxiety $\left[\operatorname{HADS} ; F_{(3,68)}=5.7, p=\right.$ 0.002 ], depression [HADS; $F_{(3,68)}=6.4, p=0.001$ ], nocturnal sleep quality [PSQI; $F_{(3,51)}=6.8, p=0.001$ ], and PTSD [IES-R; $\left.F_{(1,33)}=7.7, p=0.009\right]$.

Bonferroni-adjusted pairwise comparisons revealed no difference on any questionnaire measure between mTBI + PCS and Control + PCS participants, suggesting that their subjective symptom report was similar. This was also true for the comparison between mTBI - PCS and Control - PCS participants. The observed group differences were caused by higher symptom report in the groups with high PCS symptoms compared to those with low PCS symptoms (Table 1), as expected.

In detail, higher RPQ and CFQ symptom report was observed in $\mathrm{mTBI}+\mathrm{PCS}$ and Control + PCS participants compared to mTBI - PCS and Control - PCS (RPQ: all $p<0.001$; CFQ: all $p<0.01)$, with the exception of the comparison of CFQ score between Control + PCS and mTBI - PCS participants $(p=1.0)$. Anxiety and depression scores were higher only in Control + PCS participants compared to both mTBI - PCS (anxiety: $p=0.005$; depression; $p=0.004$ ) and Control - PCS (anxiety: $p=0.005$; depression: $p=0.003$ ). Nocturnal sleep quality was lower in mTBI + PCS participants compared to both mTBI - PCS $(p<0.001)$ and Control - PCS $(p=0.018)$ participants. Lastly, mTBI + PCS participants reported a greater number of PTSD symptoms compared to mTBI - PCS participants $(p=0.009)$.

Control + PCS participants had borderline abnormal levels of depression, but anxiety within normal bounds. Mean PSQI scores for mTBI + PCS and Control + PCS participants were indicative of poor nocturnal sleep. However, the two groups without PCS had borderline scores, suggesting a generally poor level of nocturnal sleep in the sample. All groups reported greater sleep propensity after the task compared to the beginning [KSS Post-Pre: $-\mathrm{mTBI}+$ PCS: $t_{(18)}=2.3, p=0.036$; mTBI - PCS: $t_{(18)}=2.6, p=0.020$; Control + PCS: $t_{(18)}=3.4, p=0.003$; Control - PCS: $t_{(18)}=3.6, p=0.002$ ], suggesting that the task was uniformly tiring.

\section{COGNITIVE TASKS}

\section{Error rates}

A main effect of GROUP was seen for both the n-Back $\left[F_{(3,68)}=\right.$ 8.3, $p<0.001]$ and PVSAT $\left[F_{(3,68)}=9.8, p<0.001\right]$ tasks, together with an interaction between GROUP and DIFFICULTY LEVEL for the n-Back only $\left[F_{(7,150)}=3.5, p=0.002\right.$; PVSAT: $\left.F_{(7,149)}=0.8, p=0.55\right]$. As expected, there was a main effect of DIFFICULTY LEVEL [n-Back: $F_{(2,150)}=114.3, p<0.001$; PVSAT: $\left.F_{(2,149)}=150.2, p<0.001\right]$, reflecting greater error rates with each increase in difficulty level (all comparisons: $p<0.001$, except 2 vs. $2.5: p=0.037$ ).

Bonferroni-adjusted pairwise comparisons revealed that participants with mTBI and PCS produced significantly greater error rates than all other groups (see Figure 1) for the n-Back [mTBI PCS: mean difference $(\mathrm{MD})=12.5, p<0.001$; Control + PCS: $\mathrm{MD}=11.4, p=0.001$; Control - PCS: $\mathrm{MD}=10.8$, $p=0.002]$ and the PVSAT (mTBI - PCS: MD $=15.6, p<$ 0.001; Control + PCS: $\mathrm{MD}=5.6, p<0.001$; Control - PCS: $\mathrm{MD}=11.6, p=0.005)$. All other comparisons were not statistically significant (all $p=1.0$ ).

A further series of ANOVA examined whether these GROUP differences were observed for each DIFFICULTY LEVEL in 


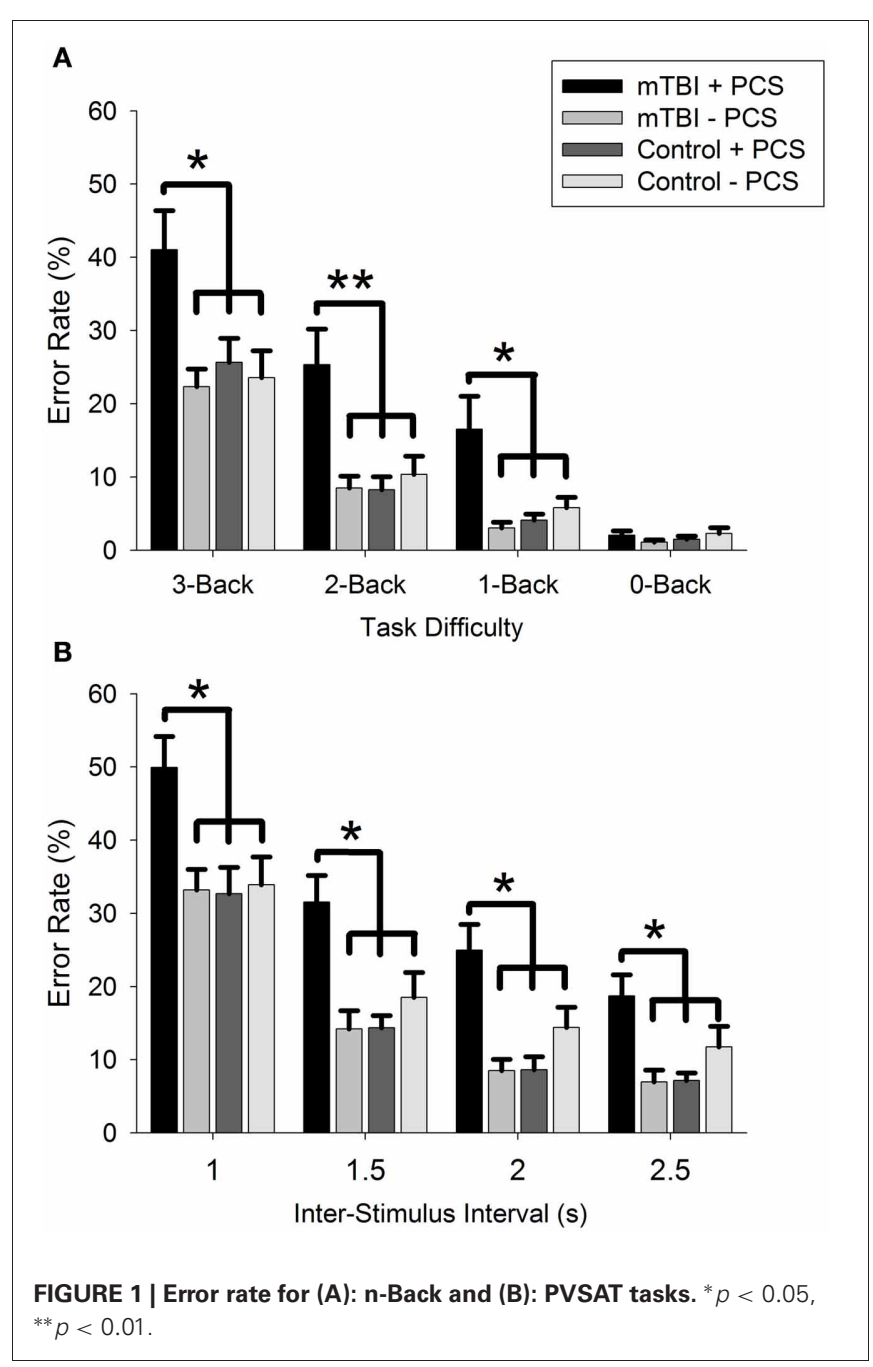

isolation. These revealed a main effect of GROUP for every condition [3-Back: $F_{(3,68)}=5.2, p=0.003 ; 2$-Back: $F_{(3,68)}=7.5$, $p<0.001$; 1-Back: $F_{(3,68)}=6.6, p=0.001 ; 1 \mathrm{~s}: F_{(3,68)}=5.2$, $p=0.003 ; 1.5 \mathrm{~s}: F_{(3,68)}=7.9, p<0.001 ; 2 \mathrm{~s}: F_{(3,68)}=9.3, p<$ $\left.0.001 ; 2.5 \mathrm{~s}: F_{(3,68)}=6.1, p=0.001\right]$, with the exception of 0 Back $\left[F_{(3,68)}=0.9, p=0.47\right]$. Again, it was the mTBI + PCS group that produced greater error rates than all other groups for the n-Back (3-Back: all $p<0.05$; 2-Back: all $p<0.005$; 1 Back: all $p<0.05$ ) and the PVSAT ( $1 \mathrm{~s}$ : all $p<0.05 ; 1.5 \mathrm{~s}$ : all $p<0.05 ; 2 \mathrm{~s}$ : all $p<0.05)$. However, in the $2.5 \mathrm{~s}$ condition, mTBI + PCS produced significantly greater error rates than mTBI - PCS $(\mathrm{MD}=11.8, p=0.002)$ and Control + PCS $(\mathrm{MD}=11.6, p=0.003)$, but not Control $-\mathrm{PCS}(\mathrm{MD}=11.6$, $p=0.18)$.

\section{Reaction time}

For both tasks, the main effect of GROUP and the GROUP $\times$ DIFFICULTY LEVEL interaction were not statistically significant (Figure 2). However, there was a main effect of DIFFICULTY LEVEL [n-Back: $F_{(2,123)}=149.7, p<0.001$; PVSAT: $F_{(2,119)}=$ 59.4, $p<0.001]$, with participants responding slower on the

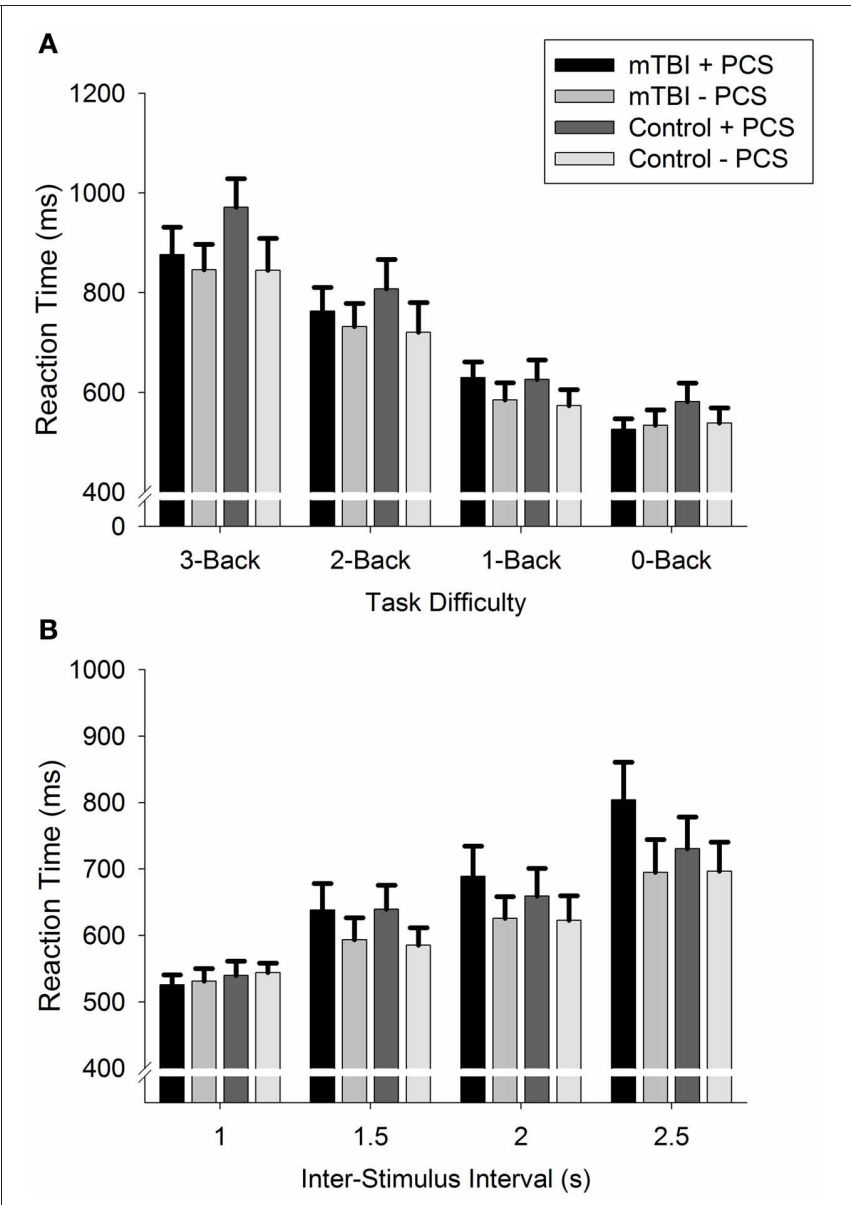

FIGURE 2 | Reaction Time for (A): n-Back and (B): PVSAT tasks.

n-Back and faster on the PVSAT as task difficulty increased (all comparisons: $p<0.001)$.

\section{CORRELATION BETWEEN SUBJECTIVE SYMPTOM REPORT AND OBJECTIVE COGNITIVE PERFORMANCE}

Correlation between greater PCS symptom report and poorer task performance was not statistically significant (after multiple comparison correction) in mTBI participants for the PVSAT task (Rho $=0.35, p=0.02$, Table 2$)$, nor the n-Back task (Rho = $0.43, p=0.004)$. Although the $n-B a c k$ association $(p=0.004)$ fell short of the significance threshold $(p=0.002)$, it represents a medium size effect according to Cohen's (Cohen, 1988) interpretation criteria, along with the PVSAT association. No significant correlations with cognitive performance were observed in control participants for any co-variable.

However, there was a significant correlation between lower sleep quality (PSQI) and poorer performance on the PVSAT task (Rho $=0.62, p<0.001$ ) for mTBI participants. There were medium size effects for correlations between poor PVSAT performance and higher anxiety (Rho $=0.44, p=0.004)$ and poor n-Back performance and high post-traumatic stress symptoms (Rho $=0.43, p=0.004)$, though these associations fell short of the significance threshold. 
Table 2 | Correlations between symptom report and cognitive task performance.

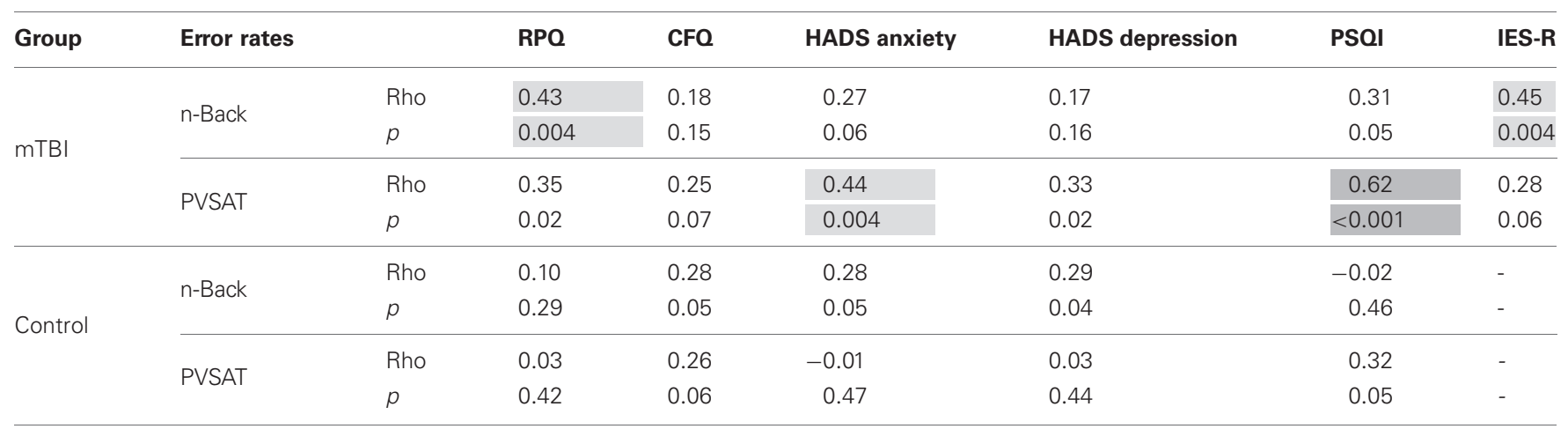

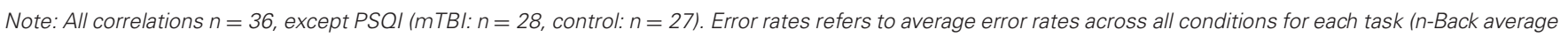

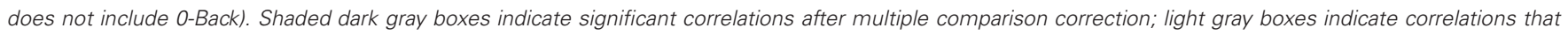
approach significance.

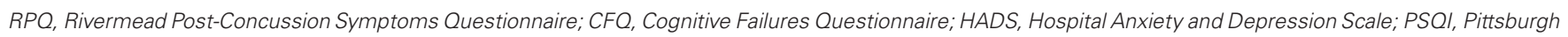
Sleepiness Index; IES-R, Impact of Event Scale-Revised.

\section{DISCUSSION \\ PRINCIPAL FINDINGS}

This study demonstrated working memory and information processing speed impairments in participants with mTBI and persistent ( $>1$ year post-injury) PCS. Cognitive performance was similar in mTBI participants without PCS and all non-head injured participants. Critically, this is despite the Control + PCS group displaying comparable subjective report of postconcussion symptoms, cognitive failures, depression, anxiety, and sleep quality to the mTBI + PCS participants. This suggests that the cognitive deficit seen in the mTBI + PCS group is not a result of high PCS symptom report per se, nor a result of the co-variables associated with PCS, but is perhaps due to the combination of ongoing PCS symptom report after initial injury. Therefore, PCS symptoms may have a differential cause, with the mechanisms leading to PCS after mTBI distinct from those contributing to the PCS symptoms seen in the general population.

Although there are some studies on cognitive performance after mTBI that have taken PCS into consideration (Chan, 2001; Wang et al., 2006; Ptito et al., 2007), there are none to our knowledge that have controlled for PCS in both mTBI and control participants. The latter allows a tentative dissociation of the effect of PCS symptom report subsequent to mTBI on cognitive performance from the influence of post-concussion-like co-variables observed in non-head injured populations.

\section{COGNITIVE TASKS}

Participants in the mTBI + PCS group were impaired on both the n-Back (working memory) and the PVSAT task (information processing speed). In contrast to our hypothesis, participants in the mTBI + PCS group were impaired on even the least cognitively demanding working memory (1-, 2-, 3- Back; Figure 1A) and information processing speed conditions $(2.5,2,1.5,1 \mathrm{~s}$ PVSAT, Figure 1B). It was assumed that the cognitive deficit would be relatively subtle, and only become apparent when task difficulty is high.
However, many previous studies have not accounted for PCS diagnosis, potentially masking cognitive impairments in a proportion of participants with mTBI. This certainly seems to be the case if the current dataset is re-analyzed without taking account of PCS (Figure 3; 2 groups: mTBI, $n=36$; Control, $n=36$ ).

Whilst there is still an overall effect of GROUP for both the n$\operatorname{Back}\left[F_{(1,70)}=4.6, p=0.036\right]$ and PVSAT $\left[F_{(1,70)}=4.7, p=\right.$ $0.034]$, there is no interaction between GROUP and DIFFICULTY LEVEL, and the GROUP difference is only significant for the 2Back $\left[F_{(1,70)}=5.4, p=0.023\right]$ and $1 \mathrm{~s}$ PVSAT $\left[F_{(1,70)}=4.5\right.$, $p=0.037]$. Therefore, not taking PCS into account leads to the more subtle results we expected, with only the more difficult levels differentiating between groups. These results suggest that accounting for PCS diagnosis may help reduce the variability inherent to $\mathrm{mTBI}$, and create more consistent results in future research.

An important aspect of the results was that all participant groups performed near ceiling on the 0-Back condition, and there was no significant difference in error rate. This condition was used as an indication of performance validity, and the result suggests this is unlikely to have significantly contributed to the differences observed for working memory and information processing speed. However, the 0-Back is not a standardized measure of effort testing, such as the Test of Memory Malingering (TOMM; Tombaugh, 1996) and Victoria Symptom Validity Test (VSVT; Slick et al., 1997), or even tests with embedded effort sensitive measures such as the Wechsler Adult Intelligence Scale (WAIS; Iverson and Tulsky, 2003) or the Repeatable Battery for the Assessment of Neuropsychological Status (RBANS; Silverberg et al., 2007). As such, it is possible that this test may not be able to detect poor effort in the symptomatic group. However, participants had no overt incentive for poor effort, as they had been screened for any litigation and on-going chronic pain. Previous studies have suggested that participants without overt incentives for poor effort only fail standardized effort tests in a small proportion of cases (Kemp et al., 2008; Pella et al., 2012). This could be due to there being no difference in effort in these groups, due 


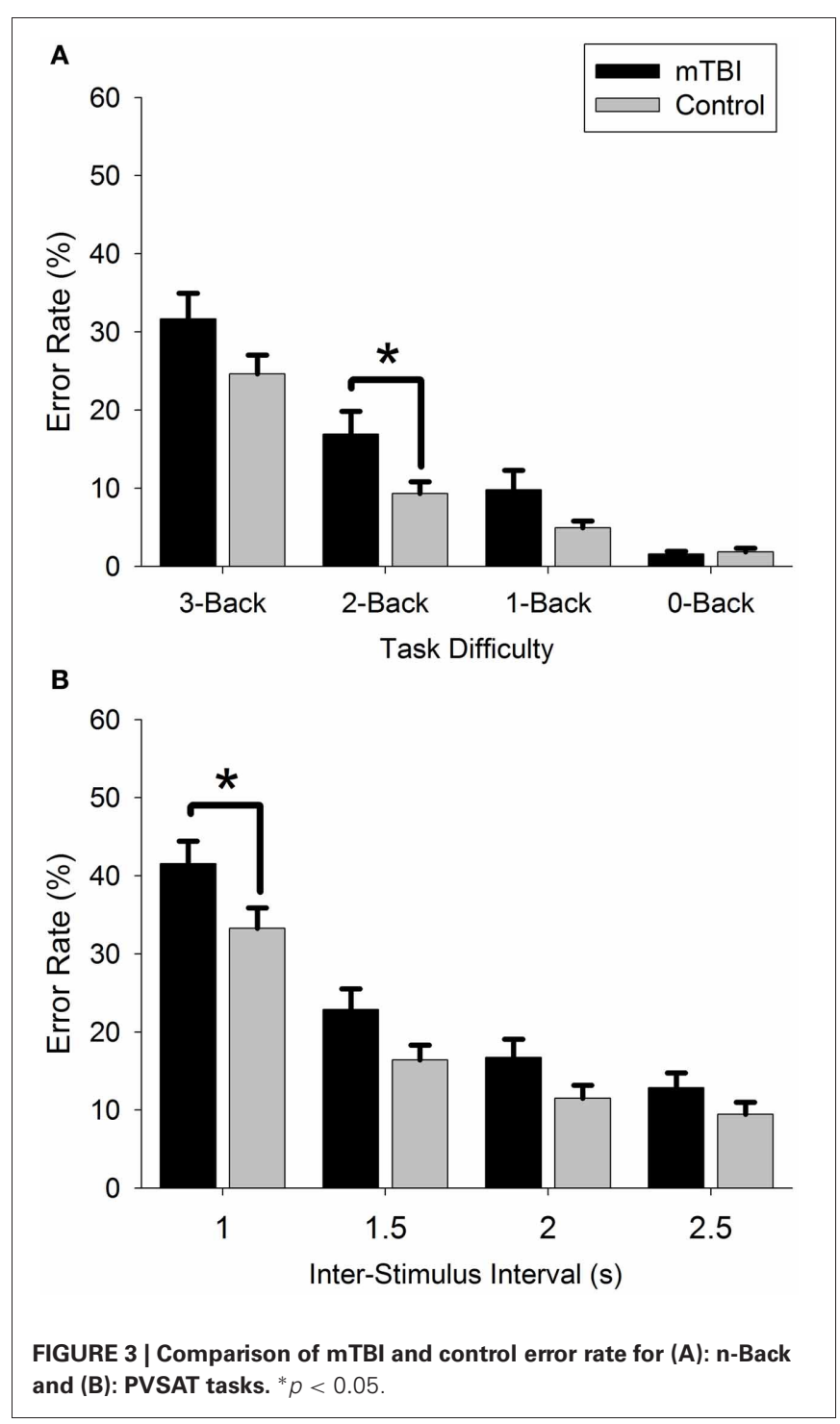

to the difference being so slight that it is not detectable, or due to the standardized tests not being suitable for detecting effort in this population.

Performance on this task could also be influenced by iatrogenic factors, such as expectation of symptoms after injury or diagnosis, leading to differences in effort. However, participants did not know whether they were in the group with or without PCS, and without such categorization participants are less likely to be influenced by iatrogenic factors in relation to PCS. Participants could be influenced by expectation of symptoms after mTBI, but both mTBI groups would be equally influenced. Therefore, if there is an effect of poor effort in this study which is not detected by the 0 -Back, then it is likely to be small, and unlikely to be the sole cause of the large deficit observed in cognitive performance.

The cognitive deficit seen in those participants with persistent PCS after mTBI may be due to a variety of underlying changes after injury. One putative mechanism which has begun to be explored is a disruption in connectivity in the default mode network (DMN; Mayer et al., 2011, 2012; Bonnelle et al., 2012; Sandrone, 2012; Sandrone and Bacigaluppi, 2012), which will need to be explored further in this participant grouping.

\section{EFFECT OF PCS AND CO-VARIABLES ON COGNITION}

The hypothesis of this study was that those participants who report persistent PCS after mTBI would have greater cognitive deficit than participants who report no long-term symptoms after mTBI. Therefore, the data was investigated to see whether increased PCS symptoms would correlate with worse cognitive performance. In addition, as PCS symptom report is influenced by other factors, such as depression, anxiety, fatigue, and posttraumatic stress, it was considered important to explore whether these co-variables correlated with cognitive performance.

\section{PCS symptoms}

There was no significant correlation between performance and PCS symptom report for either task. However, there was a medium effect size association (but one which fell short of the significance threshold) between greater PCS symptom report and poorer n-Back performance in mTBI participants $(\mathrm{Rho}=0.43$, $p=0.004$, Table 2), with no comparable association in control participants. There was also a medium effect size association for PVSAT performance and PCS symptoms in mTBI participants (Rho $=0.35, p=0.02$ ). Although these findings do not lend definitive support for a link between PCS symptoms and cognitive performance, the overall pattern of the results suggests PCS symptoms in mTBI participants may have stronger link to cognitive performance compared to control participants. This supports the hypothesis that the mechanisms leading to PCS after mTBI are distinct from those contributing to the PCS symptoms seen in the general population.

When reporting PCS symptoms using the RPQ, participants with $\mathrm{mTBI}$ are attributing the symptoms to the injury, whereas control subjects are not asked to make a specific attribution (Dean et al., 2012). It is therefore possible that an attribution bias is influencing the results, with a greater level of concern over the chronic cognitive effects of the injury causing participants with mTBI and persistent PCS to perform worse on the tasks. An attribution bias of this sort is likely to influence performance for all the cognitive tasks, as well as report of everyday cognitive failures (CFQ score). However, participants performance equally well in the 0-Back task, and CFQ score is equivalent in MTBI + PCS and control + PCS groups. An attribution bias may still be influencing the results to some extent, but not enough to explain the substantial differences seen in the working memory and information processing tasks whilst the sustained attention task (0-Back) is performed almost faultlessly. The influence of an attribution bias may be investigated further in follow-up studies being analyzed which use functional neuroimaging to look at underlying neural activity during this task.

\section{Sleep quality}

Night-time sleep quality (PSQI) was significantly worse in mTBI + PCS participants compared to both mTBI - PCS and 
Control - PCS, despite all groups having scores above or close to the threshold indicating a poor sleeper (Buysse et al., 1989). Sleep propensity (KSS) and sleepiness during the day (ESS) did not differ between the groups. It seems that although mTBI + PCS participants have poorer sleep, they do not report feeling sleepier during the daytime.

However, there was a correlation between poor PVSAT performance and poor sleep quality in mTBI participants (Rho = $0.62, p<0.001$, Table 2 ), with no comparable association in controls. This indicates that the poor sleep quality of some mTBI participants may be having an effect on aspects of their daytime functioning, even if there is no difference in reported daytime sleepiness and sleep propensity. Anecdotal evidence suggests that participants may revert to responding to all stimuli as non-targets when they felt under time pressure. This may also help to explain why there was no significant correlation between n-Back performance and sleep quality in mTBI participants ( $\mathrm{Rho}=0.31$, $p=0.05)$.

Previous studies have investigated the role of sleep in the short and long-term after mTBI (Ayalon et al., 2007; Schreiber et al., 2008; Chaput et al., 2009), and the present study confirms the association between poor sleep and long-term outcome from $\mathrm{mTBI}$. Sleep could be a risk factor for poor outcome from mTBI, with poor sleep prior to injury undermining subsequent recovery from PCS symptoms. Alternatively, the mTBI itself could trigger sleep problems in previously good sleepers, which in turn may prevent full recovery. In both cases sleep management programs provided after the initial injury could be a relatively simple treatment option to reduce long-term consequences of mTBI.

\section{Post-traumatic stress disorder}

PTSD (Bryant et al., 2009) is elevated in mTBI participants with PCS in comparison to mTBI participants without PCS. It is not possible to calculate PTSD in non-injured control participants. Therefore, we are unable to rule out the effect of PTSD on cognition, especially as the correlation between higher IES-R score and worse performance on the $n$-Back showed a medium effect size association (falling short of statistical significance; Rho $=0.43$, $p=0.004$, Table 2). Previous studies have used a control group that have sustained an injury to another part of the body without concurrent head injury (Bryant et al., 2009; Vanderploeg et al., 2009; Brenner et al., 2010a). Future studies will require a similar control group to investigate the effect of PTSD on cognitive performance in this paradigm.

\section{Depression and anxiety}

Depression and anxiety have the potential to affect both PCS symptom report and cognitive performance (Suhr and Gunstad, 2002; Iverson, 2006; Moore et al., 2006). There was no significant correlation between cognitive performance and depression in either experimental group. This is despite previous research suggesting a strong association between depression, PCS symptoms, and cognitive functioning (Suhr and Gunstad, 2002; Iverson, 2006; Sheline et al., 2010). The lack of any such effect here could be due to a difference in the sample tested (the majority studies recruit from hospitals, whereas this study recruited from a nonhosptial sample) or the depression scale used [this study used the
HADS (Bjelland et al., 2002), whereas the Beck depression inventory (Beck et al., 1961) is sometimes used elsewhere]. However, there was a medium effect size association (falling short of statistical significance) between increased anxiety and worse PVSAT performance in mTBI participants (Rho $=0.44, p=0.004)$. High anxiety in participants with $\mathrm{mTBI}$ could be related to the symptom of hypochondriacal concern as detailed in the ICD-10 criteria for PCS (WHO, 1992) [but not DSM-IV criteria (APA, 1994)]. Another possibility is that those with high anxiety may also have lower sleep quality, and it is this combination that is affecting PVSAT performance. This is an intriguing possibility, especially as there is a significant correlation between sleep quality and performance on the same task (PVSAT). Furthermore, participants in the mTBI + PCS and mTBI - PCS groups exhibited statistically different sleep quality, but no difference in anxiety levels. This requires further research, although the cognitive deficits seen in the mTBI + PCS group cannot be explained purely by increased anxiety levels as Control + PCS participants reported similar levels. Overall, it seems that the influence of anxiety on cognitive performance in this sample may be slight, and there is no tangible evidence of the influence of depression.

\section{CONCLUSION}

This study investigated the long-term ( $>1$ year) effects of mTBI on cognition, taking into account PCS in mTBI participants and PCS-like symptoms in control participants. Working memory and information processing speed was significantly impaired in mTBI participants with persistent PCS compared to mTBI participants without PCS and all non-head injured participants. Correlations between cognitive performance and symptoms were only observed for mTBI participants, with worse performance correlating with lower sleep quality, in addition to medium effect size associations (falling short of statistical significance) with higher PCS symptoms, PTSD, and anxiety.

The use of a control group with similar post-concussion symptoms to the participants with $\mathrm{mTBI}$ and PCS enabled us to distinguish to a certain extent the influence of confounders such as general cognitive failures, depression, anxiety, sleep quality, and sleepiness from the effect of the brain injury. These results suggest that the reduction in cognitive performance is not due to greater symptom report itself, but is associated to some extent with the initial injury. Furthermore, the results validate the utility of our participant grouping, and demonstrate its potential to reduce the variability observed in previous studies. However, the influence of sleep quality, and to a certain extent PTSD and anxiety, on cognitive performance requires further investigation. A longitudinal study using this protocol would be useful to elucidate the changes over time in these groups. Furthermore, some of the limitations inherent to meta-analyses of cognitive symptoms after mTBI (Pertab et al., 2009; Iverson, 2010; Rohling et al., 2011) may be alleviated using these participant groupings.

\section{ACKNOWLEDGMENTS}

This study was supported by a Wingate Foundation Scholarship, UK. We would like to thank Darragh O'Neill for his help in the preparation of this manuscript. 


\section{REFERENCES}

APA. (1994). Diagnosis and Statistical Manual of Mental Disorders. Washington, DC: American Psychiatric Association.

Ayalon, L., Borodkin, K., Dishon, L., Kanety, H., and Dagan, Y. (2007). Circadian rhythm sleep disorders following mild traumatic brain injury. Neurology 68, 1136-1140.

Bailey, C. M., Echemendia, R. J., and Arnett, P. A. (2006). The impact of motivation on neuropsychological performance in sports-related mild traumatic brain injury. J. Int. Neuropsychol. Soc. 12, 475-484.

Bazarian, J. J., McClung, J., Shah, M. N., Cheng, Y. T., Flesher, W., and Kraus, J. (2005). Mild traumatic brain injury in the United States, 1998-2000. Brain Inj. 19, 85-91.

Beck, A. T., Ward, C. H., Mendelson, M., Mock, J., and Erbaugh, J. (1961). An inventory for measuring depression. Arch. Gen. Psychiatry 4, 561-571.

Bell, B. D., Primeau, M., Sweet, J. J., and Lofland, K. R. (1999). Neuropsychological functioning in migraine headache, nonheadache chronic pain, and mild traumatic brain injury patients. Arch. Clin. Neuropsychol. 14, 389-399.

Bjelland, I., Dahl, A. A., Haug, T. T., and Neckelmann, D. (2002). The validity of the Hospital Anxiety and Depression Scale. An updated literature review. J. Psychosom. Res. 52, 69-77.

Bonnelle, V., Ham, T. E., Leech, R., Kinnunen, K. M., Mehta, M. A., Greenwood, R. J., et al. (2012). Salience network integrity predicts default mode network function after traumatic brain injury. Proc. Natl. Acad. Sci. U.S.A. 109, 4690-4695.

Braver, T. S., Cohen, J. D., Nystrom, L. E., Jonides, J., Smith, E. E., and Noll, D. C. (1997). A parametric study of prefrontal cortex involvement in human working memory. Neuroimage 5, 49-62.

Brenner, L. A., Ivins, B. J., Schwab, K., Warden, D., Nelson, L. A., Jaffee, M., et al. (2010a). Traumatic brain injury, posttraumatic stress disorder, and postconcussive symptom reporting among troops returning from iraq. J. Head Trauma Rehabil. 25, 307-312.

Brenner, L. A., Terrio, H., Homaifar, B. Y., Gutierrez, P. M., Staves, P. J., Harwood, J. E., et al. (2010b). Neuropsychological test performance in soldiers with blast-related mild TBI. Neuropsychology 24, 160-167.

Broadbent, D. E., Cooper, P. F., Fitzgerald, P., and Parkes, K. R.
(1982). The Cognitive Failures Questionnaire (CFQ) and its correlates. Br. J. Clin. Psychol. 21, 1-16.

Broglio, S. P., Pontifex, M. B., O'Connor, P., and Hillman, C. H. (2009). The persistent effects of concussion on neuroelectric indices of attention. J. Neurotrauma 26, 1463-1470.

Bryant, R. A., Creamer, M., O’Donnell, M., Silove, D., Clark, C. R., and McFarlane, A. C. (2009). Posttraumatic amnesia and the nature of post-traumatic stress disorder after mild traumatic brain injury. J. Int. Neuropsychol. Soc. 15, 862-867.

Buysse, D. J., Reynolds, C. F. 3rd., Monk, T. H., Berman, S. R., and Kupfer, D. J. (1989). The Pittsburgh Sleep Quality Index: a new instrument for psychiatric practice and research. Psychiatry Res. 28, 193-213.

Catale, C., Marique, P., Closset, A., and Meulemans, T. (2009). Attentional and executive functioning following mild traumatic brain injury in children using the Test for Attentional Performance (TAP) battery. J. Clin. Exp. Neuropsychol. 31, 331-338.

Chan, R. C. (2001). Base rate of postconcussion symptoms among normal people and its neuropsychological correlates. Clin. Rehabil. 15, 266-273.

Chan, R. C. (2005). Sustained attention in patients with mild traumatic brain injury. Clin. Rehabil. 19, 188-193.

Chaput, G., Giguere, J. F., Chauny, J. M., Denis, R., and Lavigne, G. (2009). Relationship among subjective sleep complaints, headaches, and mood alterations following a mild traumatic brain injury. Sleep Med. 10, 713-716.

Chen, J. K., Johnston, K. M., Collie, A., McCrory, P., and Ptito, A. (2007). A validation of the post concussion symptom scale in the assessment of complex concussion using cognitive testing and functional MRI. J. Neurol. Neurosurg. Psychiatry 78, 1231-1238.

Chen, J. K., Johnston, K. M., Frey, S. Petrides, M., Worsley, K., and Ptito, A. (2004). Functional abnormalities in symptomatic concussed athletes: an fMRI study. Neuroimage 22 , 68-82.

Cicerone, K. D., and Azulay, J. (2002). Diagnostic utility of attention measures in postconcussion syndrome. Clin. Neuropsychol. 16, 280-289.

Cohen, J. (1988). Statistical Power Analysis for the Behavioural Sciences, 2nd Edn. Hillsdale, NJ: Erlbaum.
Dean, P. J., O'Neill, D., and Sterr, A. (2012). Post-concussion syndrome: prevalence after mild traumatic brain injury in comparison with a sample without head injury. Brain Inj. 26, 14-26.

Dikmen, S., Machamer, J., Fann, J. R., and Temkin, N. R. (2010) Rates of symptom reporting following traumatic brain injury. J. Int Neuropsychol. Soc. 16, 401-411.

Erez, A. B., Rothschild, E., Katz, N. Tuchner, M., and Hartman-Maeir A. (2009). Executive functioning, awareness, and participation in daily life after mild traumatic brain injury: a preliminary study. Am. J. Occup. Ther. 63, 634-640.

Fear, N. T., Jones, E., Groom, M. Greenberg, N., Hull, L., Hodgetts, T. J., et al. (2009). Symptoms of postconcussional syndrome are nonspecifically related to mild traumatic brain injury in UK Armed Forces personnel on return from deployment in Iraq: an analysis of self-reported data. Psychol. Med. 39, 1379-1387.

Fos, L. A., Greve, K. W., South, M B., Mathias, C., and Benefield, H (2000). Paced Visual Serial Addition Test: an alternative measure of information processing speed. Appl. Neuropsychol. 7, 140-146.

Gillberg, M., Kecklund, G., and Akerstedt, T. (1994). Relations between performance and subjective ratings of sleepiness during a night awake. Sleep 17, 236-241.

Greiffenstein, F. M., and Baker, J. W. (2001). Comparison of premorbid and postinjury mmpi-2 profiles in late postconcussion claimants. Clin. Neuropsychol. 15, 162-170.

Hartlage, L. C., Durant-Wilson, D. and Patch, P. C. (2001). Persistent neurobehavioral problems following mild traumatic brain injury. Arch. Clin. Neuropsychol. 16, 561-570.

Hessen, E., Anderson, V., and Nestvold, K. (2008). MMPI-2 profiles 23 years after paediatric mild traumatic brain injury. Brain Inj. 22, 39-50.

Hoge, C. W., McGurk, D., Thomas, J. L., Cox, A. L., Engel, C. C., and Castro, C. A. (2008). Mild traumatic brain injury in U.S. Soldiers returning from Iraq. N. Engl. J. Med. 358 , 453-463.

Holm, L., Cassidy, J. D., Carroll, L. J., and Borg, J. (2005). Summary of the WHO collaborating centre for neurotrauma task force on mild traumatic brain injury. J. Rehabil. Med. 37, 137-141.

Iverson, G. L. (2006). Misdiagnosis of the persistent postconcussion syndrome in patients with depression. Arch. Clin. Neuropsychol. 21, 303-310.

Iverson, G. L. (2010). Mild traumatic brain injury meta-analyses can obscure individual differences. Brain Inj. 24, 1246-1255.

Iverson, G. L., and Lange, R. T. (2003). Examination of "postconcussionlike" symptoms in a healthy sample. Appl. Neuropsychol. 10, 137-144.

Iverson, G. L., and Tulsky, D. S. (2003). Detecting malingering on the WAIS-III. Unusual Digit Span performance patterns in the normal population and in clinical groups. Arch. Clin. Neuropsychol. 18, 1-9.

Jantzen, K. J., Anderson, B., Steinberg, F. L., and Kelso, J. A. (2004). A prospective functional MR imaging study of mild traumatic brain injury in college football players. Am. J. Neuroradiol. 25, 738-745.

Johansson, B., Berglund, P., and Ronnback, L. (2009). Mental fatigue and impaired information processing after mild and moderate traumatic brain injury. Brain Inj. 23, 1027-1040.

Johns, M. W. (1991). A new method for measuring daytime sleepiness: the Epworth sleepiness scale. Sleep 14, 540-545.

Kemp, S., Coughlan, A. K., Rowbottom, C., Wilkinson, K., Teggart, V., and Baker, G. (2008). The base rate of effort test failure in patients with medically unexplained symptoms. J. Psychosom. Res. 65, 319-325.

Kennedy, J. E., Leal, F. O., Lewis, J. D., Cullen, M. A., and Amador, R. R. (2010). Posttraumatic stress symptoms in OIF/OEF service members with blast-related and non-blast-related mild TBI NeuroRehabilitation 26, 223-231.

Killam, C., Cautin, R. L., and Santucci, A. C. (2005). Assessing the enduring residual neuropsychological effects of head trauma in college athletes who participate in contact sports. Arch. Clin. Neuropsychol. 20, 599-611.

King, N. S., Crawford, S., Wenden, F. J., Moss, N. E., and Wade, D. T. (1995). The Rivermead Post Concussion Symptoms Questionnaire: a measure of symptoms commonly experienced after head injury and its reliability. J. Neurol. 242, 587-592.

Kinnunen, K. M., Greenwood, R. Powell, J. H., Leech, R., Hawkins, P. C., Bonnelle, V., et al. (2011). White matter damage and cognitive impairment after traumatic brain injury. Brain 134, 449-463.

Korinthenberg, R., Schreck, J., Weser, J., and Lehmkuhl, G. (2004) 
Post-traumatic syndrome after minor head injury cannot be predicted by neurological investigations. Brain Dev. 26, 113-117.

Kumar, S., Rao, S. L., Nair, R. G., Pillai, S., Chandramouli, B. A., and Subbakrishna, D. K. (2005). Sensory gating impairment in development of post-concussive symptoms in mild head injury. Psychiatry Clin. Neurosci. 59, 466-472.

Lachapelle, J., Bolduc-Teasdale, J., Ptito, A., and McKerral, M. (2008). Deficits in complex visual information processing after mild TBI: electrophysiological markers and vocational outcome prognosis. Brain Inj. 22, 265-274.

Lange, R. T., Iverson, G. L., and Franzen, M. D. (2009). Neuropsychological functioning following complicated vs. uncomplicated mild traumatic brain injury. Brain Inj. 23, 83-91.

Lannsjo, M., Af Geijerstam, J. L., Johansson, U., Bring, J., and Borg, J. (2009). Prevalence and structure of symptoms at 3 months after mild traumatic brain injury in a national cohort. Brain Inj. 23, 213-219.

Lees-Haley, P. R., Fox, D. D., and Courtney, J. C. (2001). A comparison of complaints by mild brain injury claimants and other claimants describing subjective experiences immediately following their injury. Arch. Clin. Neuropsychol. 16, 689-695.

Lundin, A., de Boussard, C., Edman, G., and Borg, J. (2006). Symptoms and disability until 3 months after mild TBI. Brain Inj. 20, 799-806.

Mangels, J. A., Craik, F. I., Levine, B., Schwartz, M. L., and Stuss, D. T. (2002). Effects of divided attention on episodic memory in chronic traumatic brain injury: a function of severity and strategy. Neuropsychologia 40, 2369-2385.

Maruta, J., Suh, M., Niogi, S. N., Mukherjee, P., and Ghajar, J. (2010). Visual tracking synchronization as a metric for concussion screening. J. Head Trauma Rehabil. 25, 293-305.

Mayer, A. R., Mannell, M. V., Ling, J., Elgie, R., Gasparovic, C., Phillips, J. P., et al. (2009). Auditory orienting and inhibition of return in mild traumatic brain injury: a FMRI study. Hum. Brain Mapp. 30, 4152-4166.

Mayer, A. R., Mannell, M. V., Ling, J., Gasparovic, C., and Yeo, R. A. (2011). Functional connectivity in mild traumatic brain injury. Hum. Brain Mapp. 32, 1825-1835.
Mayer, A. R., Yang, Z., Yeo, R. A., Pena, A., Ling, J. M., Mannell, M. V., et al. (2012). A functional MRI study of multimodal selective attention follwing mild traumatic brain injury. Brain Imaging Behav. 6 , 343-354.

McAllister, T. W., Saykin, A. J., Flashman, L. A., Sparling, M. B., Johnson, S. C., Guerin, S. J., et al. (1999). Brain activation during working memory 1 month after mild traumatic brain injury: a functional MRI study. Neurology 53 , 1300-1308.

McAllister, T. W., Sparling, M. B., Flashman, L. A., Guerin, S. J., Mamourian, A. C., and Saykin, A. J. (2001). Differential working memory load effects after mild traumatic brain injury. Neuroimage 14, 1004-1012.

Meares, S., Shores, E. A., Taylor, A. J., Batchelor, J., Bryant, R. A., Baguley, I. J., et al. (2011). The prospective course of postconcussion syndrome: the role of mild traumatic brain injury. Neuropsychology 25 454-465.

Mittenberg, W., Digiulio, D. V., Perrin, S., and Bass, A. E. (1992). Symptoms following mild head injury: expectation as aetiology. J. Neurol. Neurosurg. Psychiatry 55 200-204.

Mittenberg, W., and Strauman, S. (2000). Diagnosis of mild head injury and the postconcussion syndrome. J. Head Trauma Rehabil. 15, 783-791.

Moore, E. L., Terryberry-Spohr, L., and Hope, D. A. (2006). Mild traumatic brain injury and anxiety sequelae: a review of the literature. Brain Inj. 20, 117-132.

Mulhern, S., and McMillan, T. M. (2006). Knowledge and expectation of postconcussion symptoms in the general population. J. Psychosom. Res. 61, 439-445.

NCIPC. (2003). Report to Congress on Mild Traumatic Brain Injury in the United States: Steps to Prevent a Serious Public Health Problem. Atlanta, GA: Centers for Disease Control and Prevention.

Nelson, H. E. (1982). National Adult Reading Test. Windsor, UK: NFERNelson.

Nelson, L. A., Yoash-Gantz, R. E., Pickett, T. C., and Campbell, T. A. (2009). Relationship between processing speed and executive functioning performance among OEF/OIF veterans: implications for postdeployment rehabilitation. J. Head Trauma Rehabil. 24, 32-40.

Nolin, P., Villemure, R., and Heroux, L. (2006). Determining long-term symptoms following mild traumatic brain injury: method of interview affects self-report. Brain Inj. 20, 1147-1154.

O’Jile, J. R., Ryan, L. M., Betz, B., ParksLevy, J., Hilsabeck, R. C., Rhudy, J. L., et al. (2006). Information processing following mild head injury. Arch. Clin. Neuropsychol. 21, 293-296.

Pare, N., Rabin, L. A., Fogel, J., and Pepin, M. (2009). Mild traumatic brain injury and its sequelae: characterisation of divided attention deficits. Neuropsychol. Rehabil. 19, 110-137.

Pella, R. D., Hill, B. D., Shelton, J. T., Elliot, E., and Gouvier, W. D. (2012). Evaluation of embedded malingering indices in a non-litigating clinical sample using control, clinical and derived groups. Arch. Clin. Neuropsychol. 27, 45-57.

Perlstein, W. M., Cole, M. A., Demery, J. A., Seignourel, P. J., Dixit, N K., Larson, M. J., et al. (2004). Parametric manipulation of working memory load in traumatic brain injury: behavioral and neural correlates. J. Int. Neuropsychol. Soc. 10 724-741.

Pertab, J. L., James, K. M., and Bigler, E. D. (2009). Limitations of mild traumatic brain injury meta-analyses. Brain Inj. 23, 498-508.

Pontifex, M. B., O'Connor, P. M. Broglio, S. P., and Hillman, C. H (2009). The association between mild traumatic brain injury history and cognitive control. Neuropsychologia 47, 3210-3216.

Ptito, A., Chen, J. K., and Johnston, K. M. (2007). Contributions of functional magnetic resonance imaging (fMRI) to sport concussion evaluation. NeuroRehabilitation 22 , 217-227.

Radanov, B. P., Dvorak, J., and Valach, L. (1992). Cognitive deficits in patients after soft tissue injury of the cervical spine. Spine 17, 127-131.

Rohling, M. L., Binder, L. M., Demakis, G. J., Larrabee, G. J., Ploetz, D. M., and Langhinrichsen-Rohling, J. (2011). A Meta-analysis of neuropsychological outcome after mild traumatic brain injury: reanalysis and reconsiderations of Binder et al. (1997), Frencham et al. (2005), and Pertab et al. (2009). Clin. Neuropsychol. 25, 608-623.

Ryan, L. M., and Warden, D. L. (2003). Post concussion syndrome. Int. Rev. Psychiatry 15, 310-316.

Sandrone, S. (2012). The brain as a crystal ball: the predictive potential of default mode network.
Front. Hum. Neurosci. 6:261. doi: 10.3389/fnhum.2012.00261

Sandrone, S., and Bacigaluppi, M. (2012). Learning from default mode network: the predictive value of resting state in traumatic brain injury. J. Neurosci. 32, 1915-1917.

Schreiber, S., Barkai, G., Gur-Hartman, T., Peles, E., Tov, N., Dolberg, O. T., et al. (2008). Long-lasting sleep patterns of adult patients with minor traumatic brain injury (mTBI) and non-mTBI subjects. Sleep Med. 9, 481-487.

Segalowitz, S. J., Bernstein, D. M., and Lawson, S. (2001). P300 eventrelated potential decrements in well-functioning university students with mild head injury. Brain Cogn. 45, 342-356.

Segalowitz, S. J., and Lawson, S. (1995). Subtle symptoms associated with self-reported mild head injury. J. Learn. Disabil. 28, 309-319.

Sheline, Y. I., Price, J. L., Yan, Z. and Mintun, M. A. (2010). Restingstate functional MRI in depression unmasks increased connectivity between networks via the dorsal nexus. Proc. Natl. Acad. Sci. U.S.A 107, 11020-11025.

Shum, D., Levin, H., and Chan, R. C. (2011). Prospective memory in patients with closed head injury: a review. Neuropsychologia 49, 2156-2165.

Sigurdardottir, S., Andelic, N., Roe, C., Jerstad, T., and Schanke, A. K. (2009). Post-concussion symptoms after traumatic brain injury at 3 and 12 months post-injury: a prospective study. Brain Inj. 23, 489-497.

Silverberg, N. D., Wertheimer, J. L. and Fichtenberg, N. L. (2007). An effort index for the Repeatable Battery For The Assessment of Neuropsychological Status (RBANS). Clin. Neuropsychol. 25, 841-854.

Slick, D. J., Hopp, G., Strauss, E., and Thompson, G. (1997). Victoria Symptom Validity Test: Professional Manual. Odessa, FL: Psychological Assessment Resources.

Slobounov, S. M., Zhang, K., Pennell, D., Ray, W., Johnson, B., and Sebastianelli, W. (2010). Functional abnormalities in normally appearing athletes following mild traumatic brain injury: a functional MRI study. Exp. Brain Res. 202, 341-354.

Solbakk, A. K., Reinvang, I., Svebak, S., Nielsen, C. S., and Sundet, K. (2005). Attention to affective pictures in closed head injury: event-related brain potentials and 
cardiac responses. J. Clin. Exp. Neuropsychol. 27, 205-223.

Spencer, R. J., Drag, L. L., Walker, S. J., and Bieliauskas, L. A. (2010). Self-reported cognitive symptoms following mild traumatic brain injury are poorly associated with neuropsychological performance in OIF/OEF veterans. J. Rehabil. Res. Dev. 47, 521-530.

Sroufe, N. S., Fuller, D. S., West, B. T., Singal, B. M., Warchausky, S. A., and Maio, R. F. (2010). Postconcussive symptoms and neurocognitive function after mild traumatic brain injury in children. Pediatrics 125, e1331-e1339.

Sterr, A., Herron, K., Hayward, C., and Montaldi, D. (2006). Are mild head injuries as mild as we think? Neurobehavioral concomitants of chronic post-concussion syndrome. BMC Neurol. 6:7. doi: 10.1186/1471-2377-6-7

Stulemeijer, M., Vos, P. E., Bleijenberg, G., and van der Werf, S. P. (2007). Cognitive complaints after mild traumatic brain injury: things are not always what they seem. J. Psychosom. Res. 63, 637-645.

Suhr, J. A., and Gunstad, J. (2002). Postconcussive symptom report: the relative influence of head injury and depression. J. Clin. Exp. Neuropsychol. 24, 981-993.

Tellier, A., Marshall, S. C., Wilson, K. G., Smith, A., Perugini, M., and Stiell, I. G. (2009). The heterogeneity of mild traumatic brain injury: Where do we stand? Brain Inj. 23, 879-887.

Tombaugh, T. (1996). Test of Memory Malingering. Toronto, ON: MultiHealth Systems.

Vanderploeg, R. D., Belanger, H. G., and Curtiss, G. (2009). Mild traumatic brain injury and posttraumatic stress disorder and their associations with health symptoms. Arch. Phys. Med. Rehabil. 90, 1084-1093.

Vanderploeg, R. D., Curtiss, G., and Belanger, H. G. (2005). Long-term neuropsychological outcomes following mild traumatic brain injury. J. Int. Neuropsychol. Soc. 11 228-236.

Wang, Y., Chan, R. C., and Deng, Y. (2006). Examination of postconcussion-like symptoms in healthy university students: relationships to subjective and objective neuropsychological function performance. Arch. Clin. Neuropsychol. 21, 339-347.

Weiss, D. S. (2007). "The impact of event scale: revised," in CrossCultural Assessment of Psychological Trauma and PTSD, eds J. P. Wilson and C. S. Tang (New York, NY: Springer US), 218-238.

WHO. (1992). International Statistical Classification of Diseases and Related Health Problems. Geneva, Switzerland: World Health Organisation.

Yang, C. C., Hua, M. S., Tu, Y. K., and Huang, S. J. (2009). Early clinical characteristics of patients with persistent post-concussion symptoms: a prospective study. Brain Inj. 23, 299-306

Conflict of Interest Statement: The authors declare that the research was conducted in the absence of any commercial or financial relationships that could be construed as a potential conflict of interest.

Received: 07 May 2012; accepted: 23 January 2013; published online: 12 February 2013

Citation: Dean PJA and Sterr A (2013) Long-term effects of mild traumatic brain injury on cognitive performance. Front. Hum. Neurosci. 7:30. doi: 10.3389/ fnhum.2013.00030

Copyright (c) 2013 Dean and Sterr. This is an open-access article distributed under the terms of the Creative Commons Attribution License, which permits use, distribution and reproduction in other forums, provided the original authors and source are credited and subject to any copyright notices concerning any third-party graphics etc. 